\title{
Meson-meson correlations in baryon-baryon and antibaryon-baryon interactions
}

\author{
K. Holinde \\ Institut für Kernphysik (Theorie), Forschungszentrum Jülich GmbH, \\ D-52425 Jülich, Germany
}

\section{ABSTRACT}

Recent work of the Jülich group about the role of meson-meson correlations in baryon-baryon and antibaryon-baryon interactions is reviewed.

\section{KEYWORDS}

Meson-meson correlations, baryon-baryon interactions, antibaryon-baryon interactions.

\section{INTRODUCTION}

Quantum chromodynamics (QCD) is the underlying theory of strong interactions, with quarks and gluons as fundamental degrees of freedom. However, in the non-perturbative region of low and J̈medium energy physics, mesons and baryons definitely keep their importance as efficient, collective degrees of freedom for a wide range of nuclear phenomena. One possibility to detect quark effects is to treat as many hadronic reactions as possible from a combined conventional viewpoint, in terms of baryons and mesons. Only by such an elaborate and consistent treatment one will be able to reliably explore the limits of the conventional picture and hopefully establish discrepancies with the empirical situation, which, in turn, can possibly be identified with explicit quark-gluon effects.

In this talk I want to demonstrate the outstanding role of meson-meson correlations in baryon-baryon interactions. First we will determine correlated $\pi \pi$ as well as $K \bar{K}$ - exchange between two baryons, which should replace the sharp mass $\sigma^{\prime}$ and $\rho$ exchange used before in the Bonn nucleon-nucleon $(N N)$ [1] and Jülich hyperon-nucleon [2] interaction models. We will then describe the effect of correlated $\pi \rho$ exchange in the $N N$ interaction, especially its implication for the (strong) $\pi N N$ formfactor and the nature of the short range $N N$ repulsion. Finally we will look at the role of both correlations in nucleon-antinucleon $(N \bar{N})$ annihilation.

\section{THE BONN NN POTENTIAL}

Starting point are baryon-baryon-meson vertex functions (Fig.1) involving not only nucleons but also $\Delta$-isobars, and various mesons below $1 \mathrm{GeV}$. The analytic structure of these couplings is (essentially) determined by the quantum numbers of particles involved at the vertex. The strength is parametrized by coupling constants; in addition form factors are included, which in principle depend on all four- 


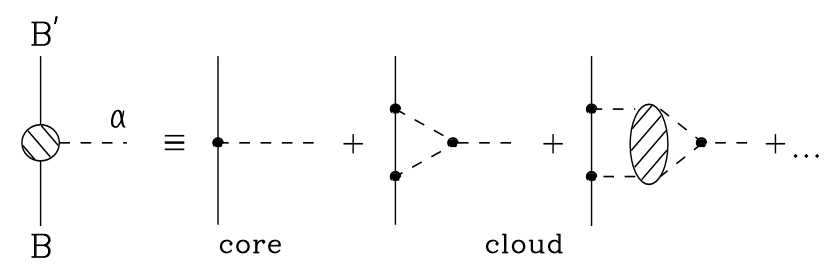

Fig.1: Baryon-baryon-meson vertex functions

These vertex functions should be considered as 'elementary' building blocks for an effective theory of nuclear reactions. As such they represent a link between QCD and nuclear physics. Ultimately therefore, QCD should determine the values of the coupling constants and the structure of the various form factors. Corresponding calculations are just beginning to emerge. For the $\pi N N$ vertex, with nucleons restricted to their mass shells, a recent QCD lattice calculation [3] yields indeed a reasonable value for $g_{N N \pi}=12.7 \pm 2.4$ and a monopole structure of the form factor with the cutoff mass $\Lambda_{N N \pi}=0.75 \pm 0.14 \mathrm{GeV}$.

In the Bonn potential [1] all form factors are parametrized in a monopole or dipole form with adjustable cutoff masses $\Lambda_{B^{\prime} B \alpha}, F_{B^{\prime} B \alpha}=\left(\frac{\Lambda^{2}-m_{\alpha}^{2}}{\Lambda^{2}+\vec{q}_{\alpha}^{2}}\right)^{n}, \mathrm{n}=1,2$. This procedure provides a total of about 10 free parameters (coupling constants and cutoff masses), which have been fixed by a fit to all $N N$ data below pion production. These vertex functions should then be used consistently in other hadronic reactions in order to explore the limits of the meson exchange concept.

Based on these vertex functions a nucleon-nucleon potential has been constructed (Fig.2) which contains apart from single meson exchanges higher order processes involving crossed two-meson exchanges and the $\Delta$-isobar in intermediate states. Correlated two-pion exchange (Fig.2d) is approximated by sharp mass $\sigma^{\prime}$ and $\rho$ exchange.

Fig.2: Processes included in the Bonn potential

The resulting description of the $N N$ data is very good; the achieved $\chi^{2}$ value per observable of less than 2 [4] certainly proves that the meson exchange picture essentially works in the low energy $N N$ system. Furthermore the Bonn potential appears to be a realistic starting point for finite nuclear calculations. Of course questions remain, e.g. about the true nature of $\omega$ exchange. It is well conceivable that $\omega$ exchange as used in the Bonn potential consists only partly of true $\omega$ exchange but parametrizes in addition different dynamics arising from higher order meson exchange diagrams and possibly explicit gluon exchange processes. We will come back to this point later.

\section{CORRELATED $\pi \pi+K \bar{K}$ EXCHANGE}

In this chapter I want to describe briefly our dynamical model [5,6] for correlated two-pion and two-kaon exchange in the baryon-baryon interaction (Fig.3), both in the scalar-isoscalar $(\sigma)$ and vector-isovector $(\rho)$ channel. The contribution of correlated $\pi \pi$ and $K \bar{K}$ exchange is derived from the amplitudes for the transition of a baryon-antibaryon state $\left(B \bar{B}^{\prime}\right)$ to a $\pi \pi$ or $K \bar{K}$ state in the pseudophysical region by applying dispersion theory and unitarity. For the $B \bar{B}^{\prime} \rightarrow \pi \pi, K \bar{K}$ amplitudes a 


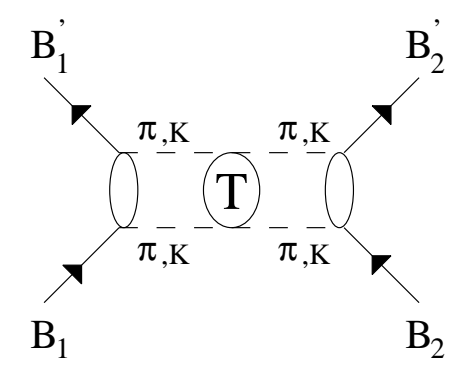

Fig.3: Correlated $\pi \pi$ and $K \bar{K}$ exchange

The Born terms include contributions from baryon exchange as well as $\rho$-pole diagrams (Fig.4). The correlations between the two pseudoscalar mesons are taken into account by means of a coupledchannel $(\pi \pi, K \bar{K})$ model $[10,11]$ generated from s- and t-channel meson exchange Born terms (Fig.5). This model describes the empirical $\pi \pi$ phase shifts over a large energy range, from threshold up to $1.3 \mathrm{GeV}$. [Quite naturally in this model, the $f_{0}(980)$ turns out to be a $K \bar{K}$ bound state. The genuine scalar resonance $\varepsilon$ has its physical mass at a much higher energy, about $1.3 \mathrm{GeV}$, and might be identified with the chiral partner of the pion.] The parameters of the $B \bar{B}^{\prime} \rightarrow \pi \pi, K \bar{K}$ model, which are related to each other by the assumption of SU(3) symmetry, are determined by the adjustment to the quasiempirical $N \bar{N} \rightarrow \pi \pi$ amplitudes in the pseudophysical region, $t \geq 4 m_{\pi}^{2}$ [7] obtained by analytic continuation of empirical $\pi N$ and $\pi \pi$ data.

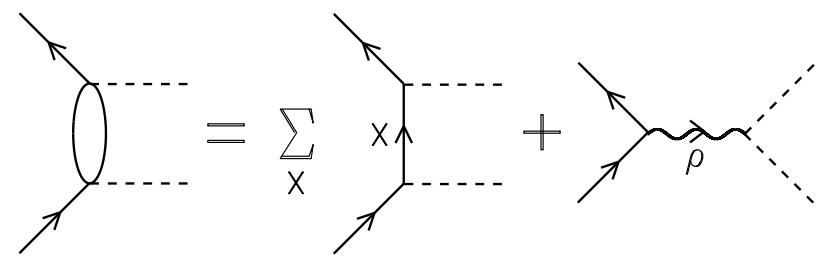

Fig.4: Microscopic model for the $B \bar{B}^{\prime} \rightarrow \pi \pi, K \bar{K}$ Born amplitudes. The solid lines denote (anti-)baryons, the dashed lines the pseudoscalar mesons $\pi \pi$ or $K \bar{K}$. The sum over exchanged baryons $X$ contains all members of the $J^{P}=\frac{1}{2}^{+}$octet and the $J^{P}=\frac{3}{2}^{+}$ decuplet which can be exchanged in accordance with the conservation of strangeness and isospin.

Schematically the baryon-baryon potential due to correlated $\pi \pi$ and $K \bar{K}$ exchanges can be represented as

$$
V_{B_{1}^{\prime}, B_{2}^{\prime} ; B_{1}, B_{2}}^{\left(0^{+}, 1^{-}\right)}(t) \sim \int_{4 m_{\pi}^{2}}^{\infty} d t^{\prime} \frac{\rho_{B_{1}^{\prime}, B_{2}^{\prime} ; B_{1}, B_{2}}^{\left(0^{+}, 1^{-}\right)}\left(t^{\prime}\right)}{t^{\prime}-t}, t<0
$$

in terms of spectral functions, which characterize both the strength and the range of the interaction. Clearly, for sharp mass exchanges, the spectral function becomes a delta-function at the appropriate mass.

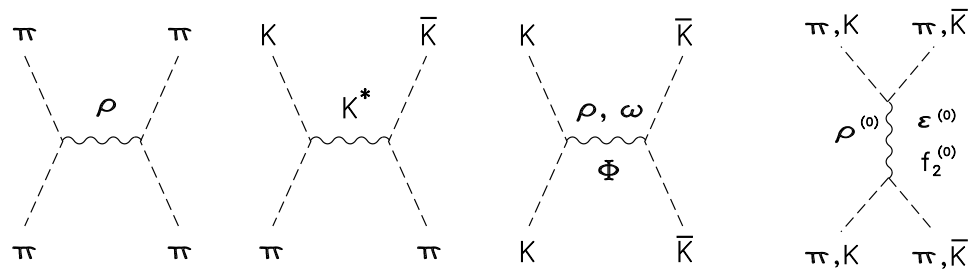

Fig.5: Born amplitudes included in the model of Refs.[10,11] for the $\pi \pi-K \bar{K}$ interaction. 
$\Sigma N \rightarrow \Sigma N$ reaction compared to sharp mass $\sigma$ exchange used in the Bonn $N N$ and Jülich $Y N$ $(Y=\Lambda, \Sigma)$ models. Obviously the $\Sigma N$ spectral function is considerably smaller than the result for the $N N$ case. Note also that correlated $K \bar{K}$ exchange processes play a minor role in the $N N$ system but are very important for the interactions involving hyperons. Compared to sharp-mass $\sigma^{\prime}$ exchange of the Bonn potential the present dynamical treatment provides stronger attraction. The difference is especially large in high partial waves since $\sigma^{\prime}$ exchange does not contain the long-ranged part of the correlated processes. As demonstrated in Ref. [5] our result indeed requires a lower $\pi N N$ coupling constant in order to accommodate the empirical high partial wave $N N$ scattering phase shifts, as suggested for quite some time by the Nijmegen group [8].

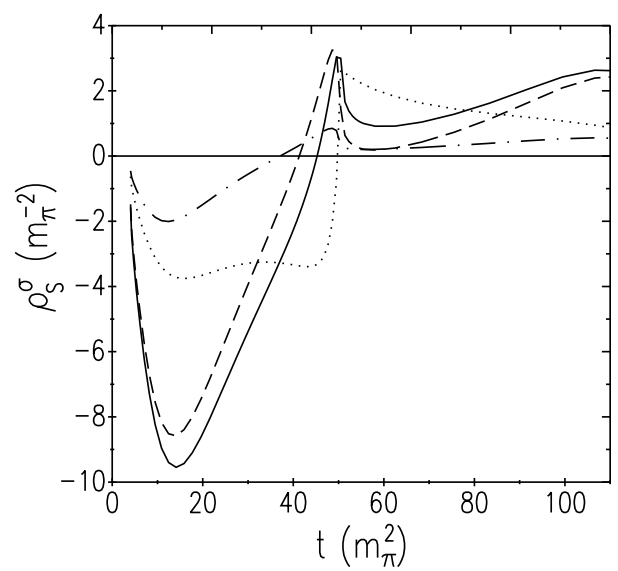

Fig.6: Spectral function $\rho_{S}^{\sigma}(t)$ in the $N N$ (solid) and $N \Sigma$ (dotted) channel derived with the full model (cf. Fig.3). If the contributions of the $B \bar{B}^{\prime} \rightarrow K \bar{K}$ Born amplitudes are neglected the dashed $(N N)$ and the dash-dotted lines $(N \Sigma)$ are obtained.

For the following discussion it is convenient to parametrize our results in terms of effective coupling strengths. In the simple one-boson-exchange picture the intermediate range attraction is provided by $\sigma$ exchange; the underlying baryon-baryon potential has the following structure

$$
V_{B_{1}^{\prime}, B_{2}^{\prime} ; B_{1}, B_{2}}^{(\sigma)}(t) \sim g_{B_{1}^{\prime} B_{1}}^{\sigma} g_{B_{2}^{\prime} B_{2}}^{\sigma} \frac{1}{m_{\sigma}^{2}-t} .
$$

The correlated potential is given by Eq. (1), which can be parametrized in terms of t-dependent strength functions $G_{B_{1}^{\prime}, B_{2}^{\prime} ; B_{1}, B_{2}}^{\left(0^{+}\right)}(t)$.

$$
V_{B_{1}^{\prime}, B_{2}^{\prime} ; B_{1}, B_{2}}^{\left(0^{+}\right)}(t) \sim G_{B_{1}^{\prime}, B_{2}^{\prime} ; B_{1}, B_{2}}^{\left(0^{+}\right)}(t) \frac{1}{m_{\sigma}^{2}-t}
$$

Note that in the OBE frame the three reactions $N N \rightarrow N N, \Sigma N \rightarrow \Sigma N, \Sigma \Sigma \rightarrow \Sigma \Sigma$ are determined by two parameters (coupling constants) $g_{N N}^{\sigma}$ and $g_{\Sigma \Sigma}^{\sigma}$ whereas the correlated exchanges are characterized by three independent strength functions, which means of course that vertex coupling constants cannot be well defined.

In the physical region the strength of the contributions is to a large extent governed by the value of $\mathrm{G}$ at $\mathrm{t}=0$. The values for the various channels (with normalization 1 in the $N N$ channel) are shown in Table 1.

Table 1: Values of the strength function $G^{\left(0^{+}\right)}(t=0)$ for $B B^{\prime}$ channels with increasing strangeness.

$\begin{array}{llllll}N N & \Lambda N & \Sigma N & \Lambda \Lambda & \Sigma \Sigma & N \Xi\end{array}$


Thus as a main result the strengths of correlated $\pi \pi$ and $K \bar{K}$ exchange decrease with increasing strangeness (becoming more negative). Furthermore they do not fulfill SU(3) relations.

The situation is similar but more complicated in the vector-isovector $(\rho)$ channel. Now we have in the OBE at each vertex a vector (g) and a tensor (f) coupling. Thus the $N N$ interaction is determined by two coupling constants, $g_{\rho N N}$ and $f_{\rho N N}$ whereas the correlated result requires three strength functions $G_{N N, N N}^{v v}(t), G_{N N, N N}^{t t}(t), G_{N N, N N}^{v t}(t)$. Fig.7 shows our results in comparison to the corresponding coupling constants including formfactors used in the Bonn potential. Again our results are considerably larger. The reason is the form factor with $\Lambda_{N N \rho}=1.4 \mathrm{GeV}$ used in the Bonn potential, which reduces the strength at $\mathrm{t}=0$ by as much as $50 \%$.
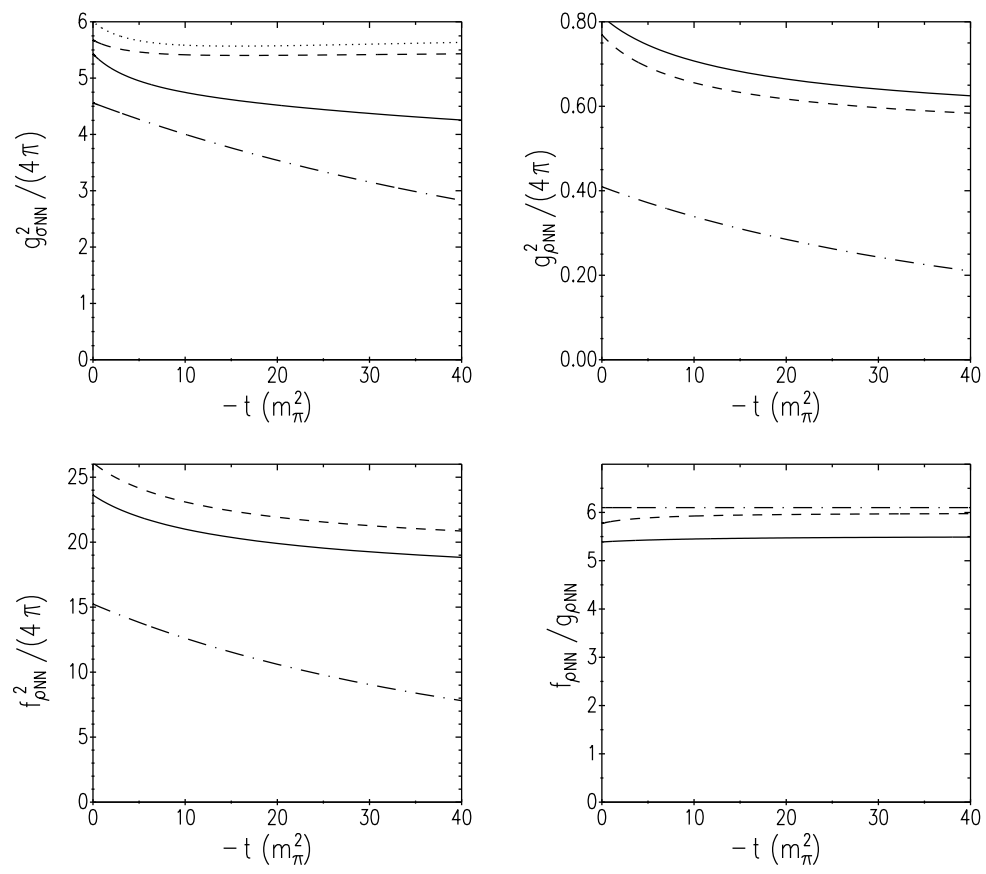

Fig.7: Effective strength of the $N N$ interaction due to correlated $\pi \pi$ and $K \bar{K}$ exchange in the $\sigma$ and $\rho$ channel as a function of the 4 -momentum transfer $t<0$. Shown are $g_{\sigma N N}^{2} \equiv G_{N N \rightarrow N N}^{\sigma}$, $g_{\rho N N}^{2} \equiv{ }^{V V} G_{N N \rightarrow N N}^{\rho}, f_{\rho N N}^{2} \equiv{ }^{T T} G_{N N \rightarrow N N}^{\rho}$ and $f_{\rho N N} / g_{\rho N N} \equiv$ $\left[{ }^{T T} G_{N N \rightarrow N N}^{\rho} /{ }^{V V} G_{N N \rightarrow N N}^{\rho}\right]^{\frac{1}{2}}$. The solid (dotted) line is derived from the microscopic model for correlated $\pi \pi$ and $K \bar{K}$ exchange using $t_{\text {max }}^{\prime}=120 m_{\pi}^{2}\left(t_{\max }^{\prime}=50 m_{\pi}^{2}\right)$ as cutoff in the dispersion integral. The dashed line follows from the quasiempirical $N \bar{N} \rightarrow \pi \pi$ amplitudes [7] with $t_{\max }^{\prime}=50 m_{\pi}^{2}$. The effective strength of $\sigma^{\prime}$ and $\rho$ exchange in the Bonn potential[1] is denoted by the dash-dotted line.

For the general baryon-baryon case, the resulting strength functions can be likewise determined. Again they deviate from what is expected in the naive $\mathrm{SU}(3)$ picture for genuine $\rho$ exchange, due to a sizable admixture of baryon exchange processes to the (SU(3) symmetric) $\rho$-pole contributions.

\section{CORRELATED $\pi \rho$ EXCHANGE}

The importance of $\rho$-exchange for the dynamics of the $N N$ system derives from the following fact: It provides a sizable intermediate range tensor force, which has opposite sign to the tensor force generated by one-pion exchange. Thus there is a strong cancellation, over a relatively broad range of energies and distances, between $\pi$ and $\rho$ exchange in the tensor channel. A similar cancellation occurs between $\mathrm{K}$ and $\mathrm{K}^{*}$ exchange, e.g. in the hyperon-nucleon interaction. Therefore, in the $N N$ (and in the baryon-baryon system in general) it is strongly suggested to always group $\pi$ and $\rho$ (as 
In fact this procedure has been an essential guideline when constructing the Bonn potential. Unfortunately it was not followed to a sufficient degree: Whereas, in second order diagrams (cp. Fig.2) $\pi \pi$ as well as $\pi \rho$ exchange has been included for uncorrelated processes (with $\mathrm{N}$ and $\Delta$ intermediate states) this has not been done for correlated processes: correlated $2 \pi$-exchange processes have been effectively included (in terms of sharp mass $\sigma^{\prime}$ and $\rho$ exchange) but correlated $\pi \rho$ processes have been left out. The reason is quite simple: The evaluation of this missing piece is technically quite complicated, much more involved (due to the spin of the $\rho$ ) compared to correlated $2 \pi$-exchange. More importantly, a dynamical model for the interaction between a $\pi$ and a $\rho$ meson was not available at that time.
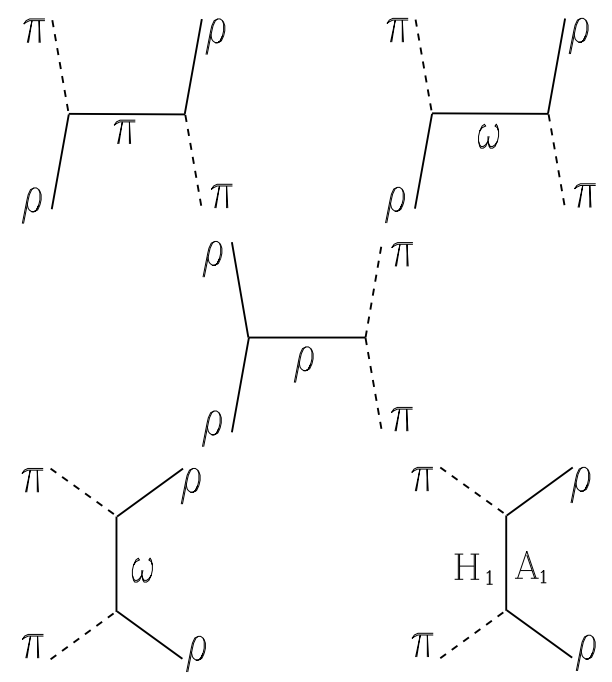

Fig.8: Born terms included in the model of Ref.[9] for the $\pi \rho$ interaction.

We have recently constructed a corresponding potential model [9], with driving terms shown in Fig.8. Open parameters have been adjusted to empirical pole parameters of the $A_{1}, H_{1}$, and $\omega$ meson.

This $\pi \rho$ T-matrix is now inserted into the correlated $\pi \rho$ exchange diagram of Fig.9. The evaluation proceeds via the same dispersion-theoretical treatment as used for the $\pi \pi$ case in the foregoing section.

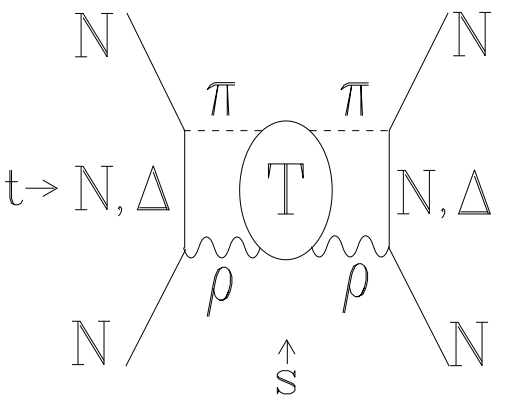

Fig.9: Correlated $\pi \rho$ exchange.

Again the result can be represented as an integral over various spectral functions in the different channels $J^{P}\left(I^{G}\right)=0^{-}\left(1^{-}\right)\left(" \pi^{\prime}\right), 1^{-}\left(0^{-}\right)\left(" \omega^{\prime}\right), 1^{+}\left(1^{-}\right)\left(" A_{1} "\right), 1^{+}\left(0^{-}\right)$(" $\left.H_{1} "\right)$ which we have considered. Thus we have for the pionic channel:

$$
V_{N, N ; N, N}^{\left(0^{-}\right)}(t) \sim \int_{\left(m_{\pi}+m_{\rho}\right)^{2}}^{\infty} d t^{\prime} \frac{\rho^{\left(0^{-}\right)}\left(t^{\prime}\right)}{t^{\prime}-t} .
$$

$\rho^{\left(0^{-}\right)}$is shown in Fig.10. Obviously it provides a sizable contribution, with a peak around $1.1 \mathrm{GeV}$, somewhat smaller than the mass $(1.2 \mathrm{GeV})$ of the phenomenological $\pi^{\prime}$ introduced in our recent $N N$ models to accommodate a soft $\pi N N$ form factor $[12]$. 


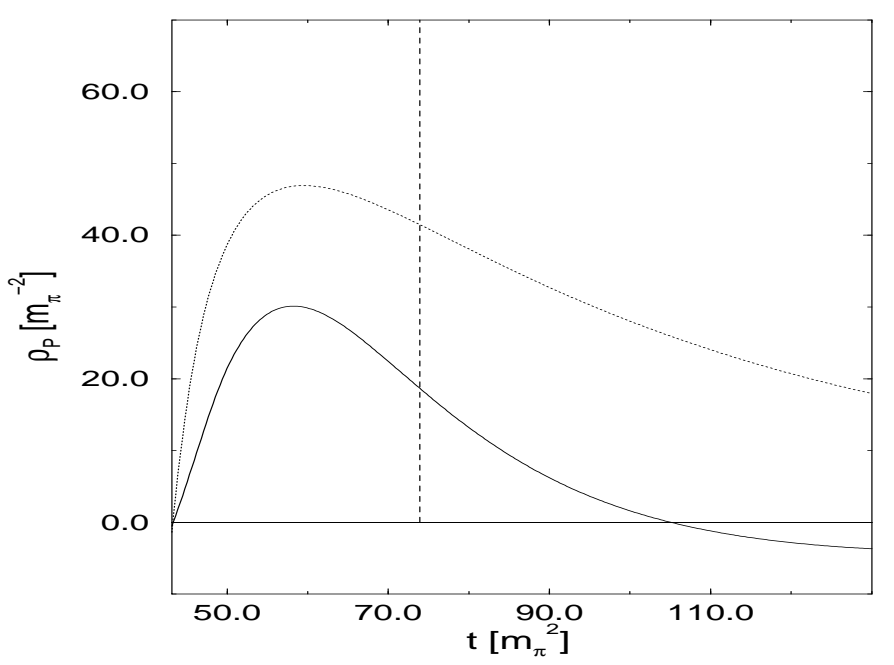

Fig.10: The spectral function in the pionic channel $\rho_{P}^{\pi}(t)$. The dotted line shows the uncorrelated part whereas the solid line represents the correlated contribution. The vertical dashed line represents sharp mass $\pi^{\prime}$ exchange used in Ref.[12].

(OPEP) when going from a cutoff mass $\Lambda_{N N \pi}$ of $1.3 \mathrm{GeV}$, phenomenologically required in the (full) Bonn potential, to a value of $1.0 \mathrm{GeV}$. For basic theoretical reasons such a reduction is highly welcome, since various information from other sources point to a rather soft $\pi N N$ form factor characterized by $\Lambda_{N N \pi} \cong 0.8 \mathrm{GeV}$ [13]. This value will probably be reached if correlated $\pi \sigma$ exchange is included, too, which is also missing in the Bonn potential. (As usual, $\sigma$ stands for a low mass correlated $\pi \pi$ pair in the $0^{+}$channel). Thus it appears that in the Bonn potential OPEP together with a hard form factor is an effective description of 'true' one-pion exchange (with a soft form factor) plus correlated $\pi \rho$ (and $\pi \sigma$ ) exchange in the pionic channel.
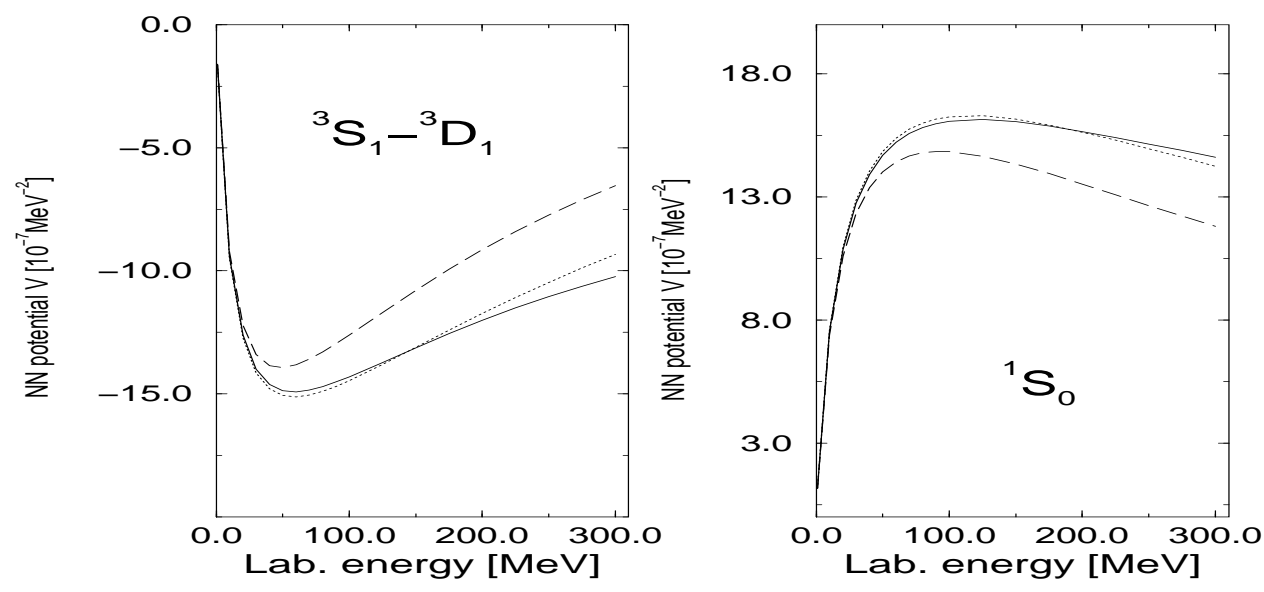

Fig.11: On-shell $N N$ potential $V_{N N}$ as function of the nucleon lab energy for the ${ }^{3} D_{1}-{ }^{3} S_{1}$ transition (lefthand side) and in the ${ }^{1} S_{0}$ state (righthand side). The dotted line denotes the one-pionexchange potential as used in the Bonn potential $\left(g_{\pi N N}^{2} / 4 \pi=\right.$ $\left.14.4, \Lambda_{\pi N N}=1.3 \mathrm{GeV}\right)$. For the dashed line, $\Lambda_{\pi N N}=1.0 \mathrm{GeV}$ was used. The solid line results if correlated $\pi \rho$ exchange in the pionic channel is added to the dashed line.

In the $\omega$ channel, the exchange of a correlated $\pi \rho$ pair also provides a sizable contribution to the $N N$ interaction. Here we have included the genuine $\omega$ meson explicitly and replaced the (effective) $\omega$ exchange in the Bonn potential by the resulting correlated $\pi \rho$ potential, which can be decomposed into a pole and a non-pole piece. The former provides a microscopic model for 'true' $\omega$ exchange leading to a renormalized $\omega N N$ coupling constant at the pole, $g_{N N \omega}^{2} / 4 \pi=11.0$, which is about a 
$N N$ interaction. It can be roughly parametrized by sharp-mass $\omega^{\prime}$-exchange with $g_{N N \omega}^{2} / 4 \pi=8.5$, $f_{N N \omega} / g_{N N \omega}=0.4$ and $m_{\omega^{\prime}}=1120 \mathrm{MeV}$.

The new reduced coupling constant (11.0) is still about a factor of 2 larger than provided by customary $\mathrm{SU}(3)$ estimates, which use $g_{N N \omega}^{2}=9 g_{N N \rho}^{2}$. Thus with $g_{N N \rho}^{2} / 4 \pi=0.55$ as determined by Hoehler and Pietarinen [14] we have $g_{N N \omega}^{2} / 4 \pi=5$. Note however, that the above relation between $\omega$ and $\rho$ coupling constants is based, apart from ideal mixing, on the assumption of vanishing $\phi N N$ coupling. For $g_{N N \phi}$ unequal to zero the above relation goes into

$$
g_{N N \omega}=3 g_{N N \rho}-\sqrt{2} g_{N N \phi} .
$$

If we take $g_{N N \phi}=-g_{N N \rho}$, (which amounts to a rather small deviation from zero) we have $g_{N N \omega}^{2} \approx$ $20 g_{N N \rho}^{2}$, in rough agreement with our results. Such a value for the $\phi N N$ coupling to the nucleon and the negative sign is quite conceivable from the $\phi$ coupling to the nucleon via the $\Lambda \bar{\Lambda}$ continuum [15].

\section{MESON-MESON CORRELATIONS IN NUCLEON-ANTINUCLEON ANNIHILATION}

We now want to turn our attention to the role of meson-meson correlations in nucleon-antinucleon annihilation. Reactions involving antinucleons have always been considered to be the ideal place for finding quark effects since annihilation phenomena from the $N \bar{N}$ system are supposedly governed by short-distance physics. Therefore the basic question is: Do these phenomena proceed via baryon exchange (Fig.12a) or via quark-gluon exchange (Fig.12b,c)?

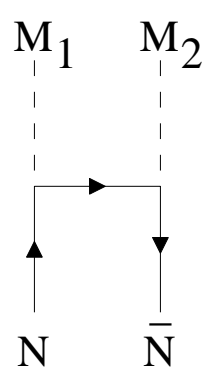

(a)

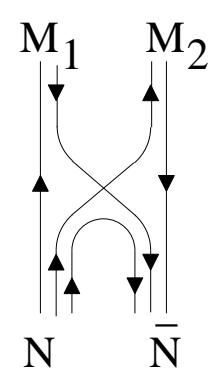

(b)

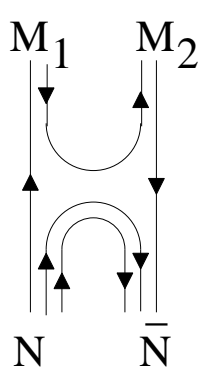

(c)

Fig.12: Baryon exchange (a) and quark-gluon $(b, c)$ transition mechanism for the annihilation process $N \bar{N} \rightarrow M_{1} M_{2}$.

As usual this question must be decided not by our personal preferences but by a serious confrontation with the empirical data. Unfortunately the data do not only see the bare transition mechanisms but also the sum of various higher order processes involving initial and final state interactions (see Fig.13). In present-day studies initial state interaction effects are usually taken into account whereas effects from final-state meson-meson correlations are in general neglected simply because not much is known about meson-meson interactions. Based on our studies of the foregoing chapters we have now the possibility to investigate the role of $\pi \pi$ (and $K \bar{K}$ ) as well as $\pi \rho$ correlations in $p \bar{p}$ annihilation.

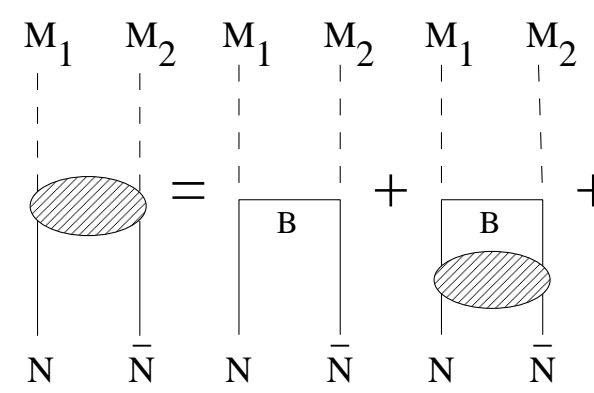

(a) (b)

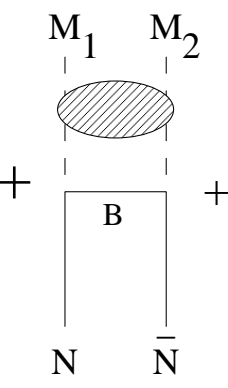

(c)

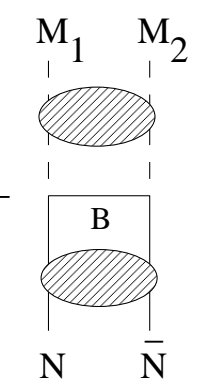

(d)

Fig.13: The transition amplitude with its contributions from the Born 


\section{$p \bar{p} \rightarrow \pi \pi, K \bar{K}, \rho \rho$}

Our calculations are based on a coupled channel model involving the channels $p \bar{p}, \pi \pi, K \bar{K}$, and $\rho \rho$. After specifying all diagonal and transition interactions one obtains in this way a simultaneous description of the elastic $(p \bar{p} \rightarrow p \bar{p})$ and annihilation $p \bar{p} \rightarrow \pi \pi, p \bar{p} \rightarrow K \bar{K}, p \bar{p} \rightarrow \rho \rho$ reactions.
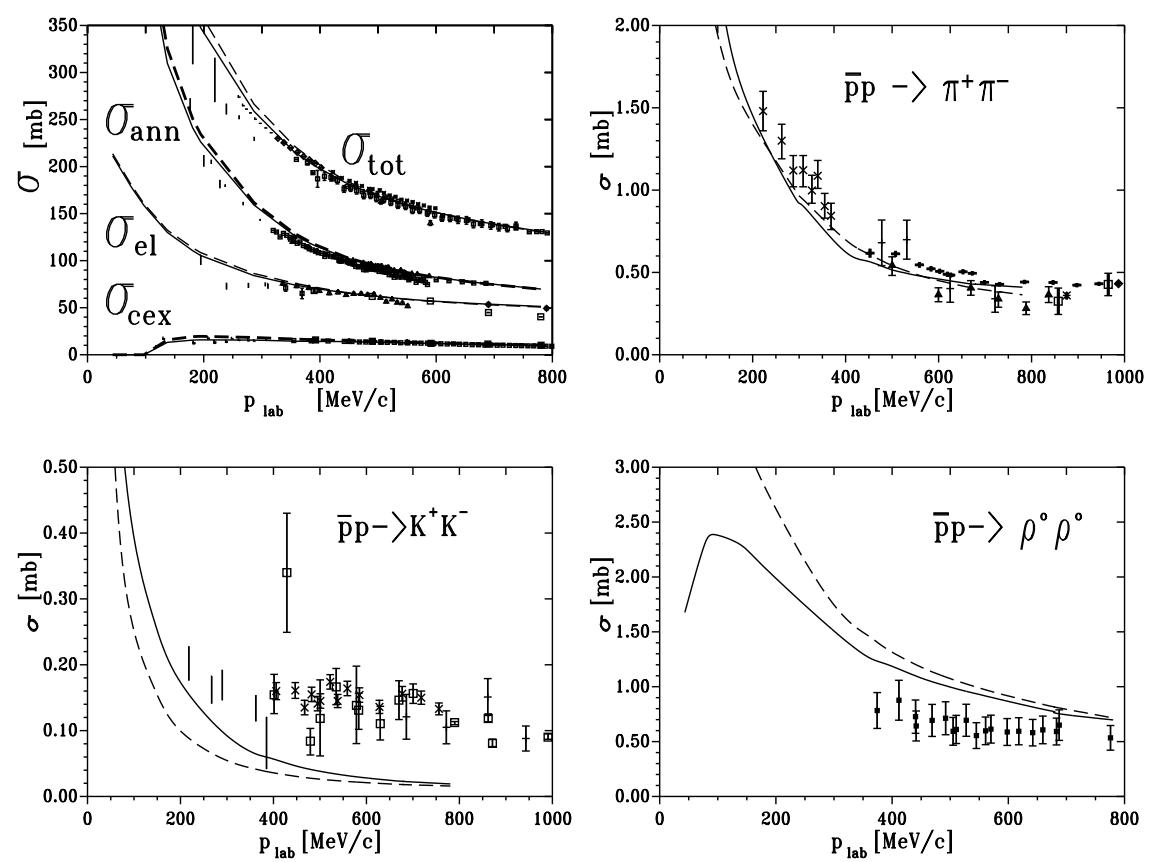

Fig.14: Cross sections for $N \bar{N}$ scattering as well as annihilation from the $N \bar{N}, \pi \pi, K \bar{K}, \rho \rho$ coupled channels system. The results of the full calculation are denoted by the solid curves. For the dashed curves, meson-meson correlations have been omitted. For references to the empirical data, see Ref.[16].

Fig.14 shows the resulting cross sections. Obviously the model gives a reasonable account of the empirical situation. Furthermore, meson- meson correlations play only a very small role. However, when we go to differential cross sections and the analyzing power for $p \bar{p} \rightarrow \pi \pi$ where empirical data are available, we notice sizable effects due to final state (meson-meson) correlations, see Fig.15.
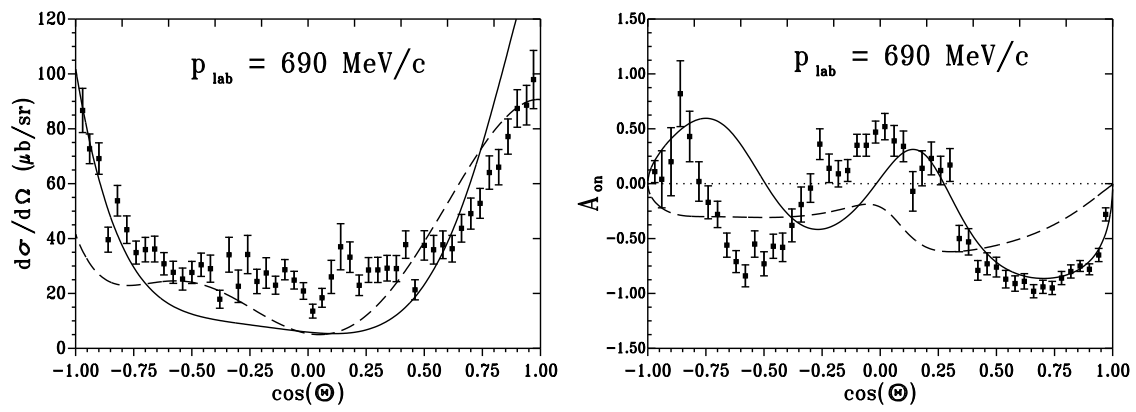

Fig.15: Differential cross section and analyzing power for the $N \bar{N} \rightarrow$ $\pi^{+} \pi^{-}$annihilation process at $p_{l a b}=690 \mathrm{MeV} / \mathrm{c}$. The curves have the same meaning as in Fig.14. For references of the empirical data, see Ref.[16].

\section{$p \bar{p} \rightarrow \pi \rho$}

Here [17] we have done a Distorted-Wave-Born-Approximation (DWBA) calculation with the initial state interaction (ISI) $p \bar{p} \rightarrow p \bar{p}$ taken from Ref. [16] and the final $\pi \rho \rightarrow \pi \rho$ interaction (FSI) from Ref. [9]. We obtain for partial cross sections from specific $p \bar{p}$ initial S-states results shown in Table 2 . The inclusion of the initial state cuts down the Born results by an order of magnitude while the inclusion 
different behaviour is just due to a different overall sign of the $\pi \rho$ amplitude in the different isospin states. Consequently final state interaction effects induce a drastic increase in the ratio, in agreement with the large empirical value, and thus are able to solve the ' $\pi \rho$ puzzle'.

Table 2: Partial cross sections for $\bar{p} p \rightarrow \pi \rho$ in $[\mu \mathrm{b}]$ at $p_{l a b}=100 \mathrm{MeV} / \mathrm{c}$.

\begin{tabular}{lrrrc}
\hline & Born & + ISI & + FSI & experiment \\
\hline $\bar{p} p\left({ }^{3} S_{1}, I=0\right)$ & 435 & 3.40 & 4.42 & \\
$\bar{p} p\left({ }^{1} S_{0}, I=1\right)$ & 126 & 0.73 & 0.14 & \\
\hline Ratio R & 3.5 & 4.7 & 32 & $35 \pm 16$ \\
\hline
\end{tabular}

\section{SUMMARY}

As a main conclusion, meson-meson correlations are important in baryon-baryon as well as antibaryon-baryon interactions and have to be included in any realistic model description.

In the baryon-baryon $(B B)$ sector, an explicit dynamical model for correlated $\pi \pi$ (plus $K \bar{K}$ ) exchange leads to results characteristically different from the simplified treatment in terms of sharp-mass $\sigma^{\prime}$ and $\rho$ exchange; for example, the effective strengths do not fulfil $\mathrm{SU}(3)$ relations. In the scalar-isoscalar channel the predicted attraction decreases with increasing strangeness (becoming more negative) of the $B B$ system.

Furthermore a model for correlated $\pi \rho$ exchange between two nucleons has been discussed, which has substantial effects: In the pionic channel it counterbalances the suppression generated by a soft $\pi N N$ form factor of monopole type with a cutoff mass of about $1 \mathrm{GeV}$; in the $\omega$-channel it provides a strong repulsive contribution, leaving little room for explicit quark-gluon effects.

The same meson-meson correlations have been discussed in the $N \bar{N}$ sector. They have substantial effects in $p \bar{p} \rightarrow \pi \pi$ spin observables and $p \bar{p} \rightarrow \pi \rho$ partial cross sections, in both cases improving the agreement with experiment. Therefore such correlations have to be included before the relevant transition mechanisms (baryon exchange on one hand and quark rearrangement or quark annihilation on the other hand) can be reliably identified.

\section{REFERENCES}

[1] R. Machleidt, K. Holinde, and Ch. Elster, (1987). Phys. Rep., 149, 1.

[2] B. Holzenkamp, K. Holinde, and J. Speth, (1989). Nucl. Phys., A500, 485.

A. Reuber, K. Holinde, and J. Speth, (1994). Nucl. Phys., A570, 543.

[3] K.F. Liu, S.J. Dong, and W. Wilcox, (1995). Phys. Rev. Lett., 74, 2172.

[4] J. Haidenbauer and K. Holinde, (1989). Phys. Rev., C40, 2465.

[5] H.-C. Kim, J. W. Durso, and K. Holinde, (1994). Phys. Rev., C49, 2355.

[6] A. Reuber, (1995). Berichte des Forschungszentrums Jülich, Nr. 3117.

A. Reuber, K. Holinde, H.-C. Kim, and J. Speth, Correlated $\pi \pi$ and $K \bar{K}$ Exchange in the Baryon-Baryon Interaction, submitted to Nucl. Phys., A.

[7] G. Höhler, F. Kaiser, R. Koch, and E. Pietarinen, (1979). "Handbook of Pion-Nucleon Scattering", Phys. Data 12-1, Fachinformationszentrum, Karlsruhe

O. Dumbrajs et al., (1983). "Compilation of Coupling Constants and Low-Energy Parameters", Nucl. Phys., B216, 277.

[8] R.A.M. Klomp, V.G.J. Stoks, and J.J. de Swart, (1991). Phys. Rev., C44, R1258.

[9] G. Janssen, K. Holinde, and J. Speth, (1994). Phys. Rev., C49, 2763.

[10] D. Lohse, J.W. Durso, K. Holinde, and J. Speth, (1990). Nucl. Phys., A516, 513.

[11] C. Schuetz, K. Holinde, J. Speth, B.C. Pearce, and J.W. Durso, (1990). Phys. Rev., C51, 1374.

[12] J. Haidenbauer, K. Holinde, and A. W. Thomas, (1994). Phys. Rev., C49, 2331.

[13] S.A. Coon, and M.D. Scadron, (1990). Phys. Rev., D42, 2256.

[14] G. Höhler and E. Pietarinen, (1975). Nucl. Phys., B95, 210.

[15] V. Mull, privat communication. 

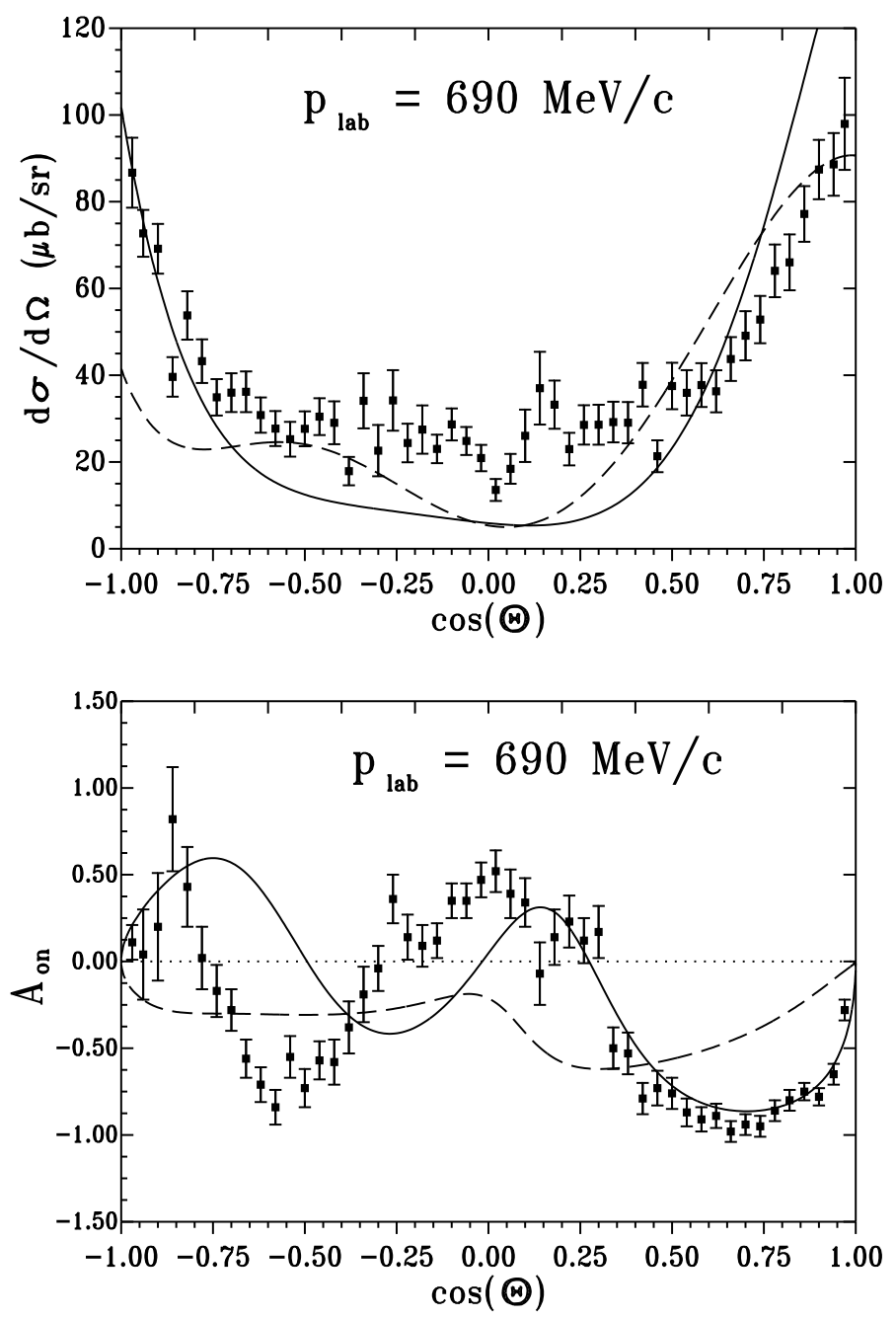